\title{
On modified Dunkl generalization of Szász operators via $q$-calculus
}

\author{
M Mursaleen ${ }^{1,2^{*}}$, Md Nasiruzzaman $^{1}$ and Abdullah Alotaibi ${ }^{2}$
}

\author{
"Correspondence: \\ mursaleenm@gmail.com \\ 'Department of Mathematics, \\ Aligarh Muslim University, Aligarh, \\ 202002, India \\ ${ }^{2}$ Operator Theory and Applications \\ Research Group, Department of \\ Mathematics, Faculty of Science, \\ King Abdulaziz University, P.O. Box \\ 80203, Jeddah, 21589, Saudi Arabia
}

\begin{abstract}
The purpose of this paper is to introduce a modification of $q$-Dunkl generalization of exponential functions. These types of operators enable better error estimation on the interval $\left[\frac{1}{2}, \infty\right)$ than the classical ones. We obtain some approximation results via a well-known Korovkin-type theorem and a weighted Korovkin-type theorem. Further, we obtain the rate of convergence of the operators for functions belonging to the Lipschitz class.
\end{abstract}

MSC: $41 \mathrm{~A} 25 ; 41 \mathrm{~A} 36 ; 33 \mathrm{C} 45$

Keywords: q-integers; Dunkl analogue; Szász operator; q-Szász-Mirakjan-Kantorovich; modulus of continuity; Peetre's K-functional

\section{Introduction and preliminaries}

In 1912, Bernstein [1] introduced the following sequence of operators $B_{n}: C[0,1] \rightarrow C[0,1]$ defined by

$$
B_{n}(f ; x)=\sum_{k=0}^{n}\left(\begin{array}{l}
n \\
k
\end{array}\right) x^{k}(1-x)^{n-k} f\left(\frac{k}{n}\right), \quad x \in[0,1]
$$

for $n \in \mathbb{N}$ and $f \in C[0,1]$.

In 1950, for $x \geq 0$, Szász [2] introduced the operators

$$
S_{n}(f ; x)=e^{-n x} \sum_{k=0}^{\infty} \frac{(n x)^{k}}{k !} f\left(\frac{k}{n}\right), \quad f \in C[0, \infty) .
$$

In the field of approximation theory, the application of $q$-calculus emerged as a new area. The first $q$-analogue of well-known Bernstein polynomials was introduced by Lupaş by applying the idea of $q$-integers [3]. In 1997, Phillips [4] considered another $q$-analogue of the classical Bernstein polynomials. Later on, many authors introduced $q$-generalizations of various operators and investigated several approximation properties [5-14].

We now present some basic definitions and notations of the $q$-calculus which are used in this paper [15].

Definition 1.1 For $|q|<1$, the $q$-number $[\lambda]_{q}$ is defined by

$$
[\lambda]_{q}= \begin{cases}\frac{1-q^{\lambda}}{1-q} & (\lambda \in \mathbb{C}), \\ \sum_{k=0}^{n-1} q^{k}=1+q+q^{2}+\cdots+q^{n-1} & (\lambda=n \in \mathbb{N}) .\end{cases}
$$

\section{Springer}


Definition 1.2 For $|q|<1$, the $q$-factorial $[n]_{q}$ ! is defined by

$$
[n]_{q} != \begin{cases}1 & (n=0), \\ \prod_{k=1}^{n}[k]_{q} & (n \in \mathbb{N}) .\end{cases}
$$

Our investigation is to construct a linear positive operator generated by a generalization of the exponential function defined by (see [16])

$$
e_{\mu}(x)=\sum_{n=0}^{\infty} \frac{x^{n}}{\gamma_{\mu}(n)}
$$

where

$$
\gamma_{\mu}(2 k)=\frac{2^{2 k} k ! \Gamma\left(k+\mu+\frac{1}{2}\right)}{\Gamma\left(\mu+\frac{1}{2}\right)},
$$

and

$$
\gamma_{\mu}(2 k+1)=\frac{2^{2 k+1} k ! \Gamma\left(k+\mu+\frac{3}{2}\right)}{\Gamma\left(\mu+\frac{1}{2}\right)} .
$$

The recursion formula for $\gamma_{\mu}$ is given by

$$
\gamma_{\mu}(k+1)=\left(k+1+2 \mu \theta_{k+1}\right) \gamma_{\mu}(k), \quad k=0,1,2, \ldots
$$

where $\mu>-\frac{1}{2}$ and

$$
\theta_{k}= \begin{cases}0 & \text { if } k \in 2 \mathbb{N} \\ 1 & \text { if } k \in 2 \mathbb{N}+1\end{cases}
$$

Sucu [17] defined a Dunkl analogue of Szász operators via a generalization of the exponential function [16] as follows:

$$
S_{n}^{*}(f ; x):=\frac{1}{e_{\mu}(n x)} \sum_{k=0}^{\infty} \frac{(n x)^{k}}{\gamma_{\mu}(k)} f\left(\frac{k+2 \mu \theta_{k}}{n}\right),
$$

where $x \geq 0, f \in C[0, \infty), \mu \geq 0, n \in \mathbb{N}$.

Cheikh et al. [18] stated the $q$-Dunkl classical $q$-Hermite-type polynomials and gave definitions of $q$-Dunkl analogues of exponential functions and recursion relations for $\mu>$ $-\frac{1}{2}$ and $0<q<1$,

$$
\begin{aligned}
& e_{\mu, q}(x)=\sum_{n=0}^{\infty} \frac{x^{n}}{\gamma_{\mu, q}(n)}, \quad x \in[0, \infty), \\
& E_{\mu, q}(x)=\sum_{n=0}^{\infty} \frac{q^{\frac{n(n-1)}{2}} x^{n}}{\gamma_{\mu, q}(n)}, \quad x \in[0, \infty),
\end{aligned}
$$


where

$$
\gamma_{\mu, q}(n)=\frac{\left(q^{2 \mu+1}, q^{2}\right)_{\left[\frac{n+1}{2}\right]}\left(q^{2}, q^{2}\right)_{\left[\frac{n}{2}\right]}}{(1-q)^{n}}, \quad n \in \mathbb{N} .
$$

Some of the special cases of $\gamma_{\mu, q}(n)$ are defined as follows:

$$
\begin{aligned}
& \gamma_{\mu, q}(0)=1, \quad \gamma_{\mu, q}(1)=\frac{1-q^{2 \mu+1}}{1-q}, \\
& \gamma_{\mu, q}(2)=\left(\frac{1-q^{2 \mu+1}}{1-q}\right)\left(\frac{1-q^{2}}{1-q}\right), \\
& \gamma_{\mu, q}(3)=\left(\frac{1-q^{2 \mu+1}}{1-q}\right)\left(\frac{1-q^{2}}{1-q}\right)\left(\frac{1-q^{2 \mu+3}}{1-q}\right), \\
& \gamma_{\mu, q}(4)=\left(\frac{1-q^{2 \mu+1}}{1-q}\right)\left(\frac{1-q^{2}}{1-q}\right)\left(\frac{1-q^{2 \mu+3}}{1-q}\right)\left(\frac{1-q^{4}}{1-q}\right) .
\end{aligned}
$$

In [19], Içöz and Çekim gave the Dunkl generalization of Szász operators via $q$-calculus as follows:

$$
D_{n, q}(f ; x)=\frac{1}{e_{\mu, q}\left([n]_{q} x\right)} \sum_{k=0}^{\infty} \frac{\left([n]_{q} x\right)^{k}}{\gamma_{\mu, q}(k)} f\left(\frac{1-q^{2 \mu \theta_{k}+k}}{1-q^{n}}\right)
$$

for $\mu>\frac{1}{2}, x \geq 0,0<q<1$ and $f \in C[0, \infty)$.

Previous studies demonstrate that providing a better error estimation for positive linear operators plays an important role in approximation theory, which allows us to approximate much faster to the function being approximated.

Motivated essentially by Içöz and Çekim's [19] recent investigation of Dunkl generalization of Szász-Mirakjan operators via $q$-calculus, we show that our modified operators have better error estimation than those in [19]. We also prove several approximation results and successfully extend the results of [19]. Several other related results are also discussed.

\section{Construction of operators and moments estimation}

Let $\left\{r_{[n]_{q}}\right\}$ be a sequence of real-valued continuous functions defined on $[0, \infty)$ with $0 \leq$ $r_{[n]_{q}}(x)<\infty$ such that

$$
r_{[n]_{q}}(x)=x-\frac{1}{2[n]_{q}}, \quad \text { where } \frac{1}{2 n} \leq x<\frac{1}{1-q^{n}} \text { and } n \in \mathbb{N} \text {. }
$$

Then, for any $\frac{1}{2 n} \leq x<\frac{1}{1-q^{n}}, 0<q<1, \mu>\frac{1}{2 n}$ and $n \in \mathbb{N}$, we define

$$
D_{n, q}^{*}(f ; x)=\frac{1}{e_{\mu, q}\left([n]_{q} r_{[n]_{q}}(x)\right)} \sum_{k=0}^{\infty} \frac{\left([n]_{q} r_{[n]_{q}}(x)\right)^{k}}{\gamma_{\mu, q}(k)} f\left(\frac{1-q^{2 \mu \theta_{k}+k}}{1-q^{n}}\right),
$$

where $e_{\mu, q}(x), \gamma_{\mu, q}$ are defined in (1.6), (1.8) by [17] and $f \in C_{\zeta}[0, \infty)$ with $\zeta \geq 0$ and

$$
C_{\zeta}[0, \infty)=\left\{f \in C[0, \infty):|f(t)| \leq M(1+t)^{\zeta} \text { for some } M>0, \zeta>0\right\}
$$


Lemma 2.1 Let $D_{n, q}^{*}(\cdot ; \cdot)$ be the operators given by (2.2). Then, for each $\frac{1}{2 n} \leq x<\frac{1}{1-q^{n}}, n \in \mathbb{N}$, we have the following identities/estimates:

(1) $D_{n, q}^{*}(1 ; x)=1$,

(2) $D_{n, q}^{*}(t ; x)=x-\frac{1}{2[n]_{q}}$,

(3) $x^{2}+\left(q^{2 \mu}[1-2 \mu]_{q} \frac{e_{\mu, q}\left(q[n]_{q} r_{[n]_{q}}(x)\right)}{e_{\mu, q}\left([n]_{q} r[n]_{q}(x)\right)}-1\right) \frac{x}{[n]_{q}}-\frac{1}{4[n]_{q}^{2}}\left(2 q^{2 \mu}[1-2 \mu]_{q} \frac{e_{\mu, q}\left(q[n]_{q} r_{[n]_{q}}(x)\right)}{e_{\mu, q}\left([n]_{q} r[n]_{q}(x)\right)}-1\right) \leq$ $D_{n, q}^{*}\left(t^{2} ; x\right) \leq x^{2}+\left([1+2 \mu]_{q}-1\right) \frac{x}{[n]_{q}}-\frac{1}{4[n]_{q}^{2}}\left(2[1+2 \mu]_{q}-1\right)$.

Proof As $D_{n, q}^{*}(1 ; x)=\frac{1}{e_{\mu, q}\left([n]_{q} r_{[n] q}(x)\right)} \sum_{k=0}^{\infty} \frac{\left([n]_{q} r_{[n]_{q}}(x)\right)^{k}}{\gamma_{\mu}(k)}=1$, and

$$
\begin{aligned}
D_{n, q}^{*}(t ; x) & =\frac{1}{e_{\mu, q}\left([n]_{q} r_{[n]_{q}}(x)\right)} \sum_{k=0}^{\infty} \frac{\left([n]_{q} r_{[n]_{q}}(x)\right)^{k}}{\gamma_{\mu}(k)}\left(\frac{1-q^{2 \mu \theta_{k}+k}}{1-q^{n}}\right) \\
& =\frac{1}{[n]_{q} e_{\mu, q}\left([n]_{q} r_{[n]_{q}}(x)\right)} \sum_{k=1}^{\infty} \frac{\left([n]_{q} r_{[n]_{q}}(x)\right)^{k}}{\gamma_{\mu}(k-1)} \\
& =x-\frac{1}{2[n]_{q}},
\end{aligned}
$$

then (1) and (2) hold. Similarly,

$$
\begin{aligned}
D_{n, q}^{*}\left(t^{2} ; x\right) & =\frac{1}{e_{\mu, q}\left([n]_{q} r_{[n]_{q}}(x)\right)} \sum_{k=0}^{\infty} \frac{\left([n]_{q} r_{[n]_{q}}(x)\right)^{k}}{\gamma_{\mu}(k)}\left(\frac{1-q^{2 \mu \theta_{k}+k}}{1-q^{n}}\right)^{2} \\
& =\frac{1}{[n]_{q}^{2} e_{\mu, q}\left([n]_{q} r_{[n]_{q}}(x)\right)} \sum_{k=0}^{\infty} \frac{\left([n]_{q} r_{[n]_{q}}(x)\right)^{k}}{\gamma_{\mu}(k-1)}\left(\frac{1-q^{2 \mu \theta_{k}+k}}{1-q}\right) \\
& =\frac{1}{[n]_{q}^{2} e_{\mu, q}\left([n]_{q} r_{[n]_{q}}(x)\right)} \sum_{k=0}^{\infty} \frac{\left([n]_{q} r_{[n]_{q}}(x)\right)^{k+1}}{\gamma_{\mu}(k)}\left(\frac{1-q^{2 \mu \theta_{k+1}+k+1}}{1-q}\right) .
\end{aligned}
$$

From [19] we know that

$$
\left[2 \mu \theta_{k+1}+k+1\right]_{q}=\left[2 \mu \theta_{k}+k\right]_{q}+q^{2 \mu \theta_{k}+k}\left[2 \mu(-1)^{k}+1\right]_{q} .
$$

Now, by separating to the even and odd terms and using (2.4), we get

$$
\begin{aligned}
D_{n, q}^{*}\left(t^{2} ; x\right)= & \frac{1}{[n]_{q}^{2} e_{\mu, q}\left([n]_{q} r_{[n]_{q}}(x)\right)} \sum_{k=0}^{\infty} \frac{\left([n]_{q} r_{[n]_{q}}(x)\right)^{k+1}}{\gamma_{\mu}(k)}\left(\frac{1-q^{2 \mu \theta_{k+1}+k+1}}{1-q}\right) \\
& +\frac{[1+2 \mu]_{q}}{[n]_{q}^{2} e_{\mu, q}\left([n]_{q} r_{[n]_{q}}(x)\right)} \sum_{k=0}^{\infty} \frac{\left([n]_{q} r_{[n]_{q}}(x)\right)^{2 k+1}}{\gamma_{\mu}(2 k)} q^{2 \mu \theta_{2 k+2 k}} \\
& +\frac{[1-2 \mu]_{q}}{[n]_{q}^{2} e_{\mu, q}\left([n]_{q} r_{[n]_{q}}(x)\right)} \sum_{k=0}^{\infty} \frac{\left([n]_{q} r_{[n]_{q}}(x)\right)^{2 k+2}}{\gamma_{\mu}(2 k)} q^{2 \mu \theta_{2 k+1}+2 k+1} .
\end{aligned}
$$

Since

$$
[1-2 \mu]_{q} \leq[1+2 \mu]_{q}
$$


we have

$$
\begin{aligned}
D_{n, q}^{*}\left(t^{2} ; x\right) \geq & \left(r_{[n]_{q}}(x)\right)^{2}+\frac{r_{[n]_{q}}(x)[1-2 \mu]_{q}}{[n]_{q} e_{\mu, q}\left([n]_{q} r_{[n]_{q}}(x)\right)} \sum_{k=0}^{\infty} \frac{\left(q[n]_{q} r_{[n]_{q}}(x)\right)^{2 k}}{\gamma_{\mu}(2 k)} \\
& +\frac{q^{2 \mu} r_{[n]_{q}}(x)[1-2 \mu]_{q}}{[n]_{q} e_{\mu, q}\left([n]_{q} r_{[n]_{q}}(x)\right)} \sum_{k=0}^{\infty} \frac{\left(q[n]_{q} r_{[n]_{q}}(x)\right)^{2 k+1}}{\gamma_{\mu}(2 k+1)} \\
\geq & \left(r_{[n]_{q}}(x)\right)^{2}+q^{2 \mu}[1-2 \mu]_{q} \frac{e_{\mu, q}\left(q[n]_{q} r_{[n]_{q}}(x)\right)}{e_{\mu, q}\left([n]_{q} r_{[n]_{q}}(x)\right)} \frac{r_{[n]_{q}}(x)}{[n]_{q}}
\end{aligned}
$$

On the other hand, we have

$$
D_{n, q}^{*}\left(t^{2} ; x\right) \leq\left(r_{[n]_{q}}(x)\right)^{2}+[1+2 \mu]_{q} \frac{r_{[n]_{q}}(x)}{[n]_{q}} .
$$

This completes the proof.

Lemma 2.2 Let the operators $D_{n, q}^{*}(\cdot ; \cdot)$ be given by $(2.2)$. Then, for each $x \geq \frac{1}{2 n}, n \in \mathbb{N}$, we have

(1) $D_{n, q}^{*}(t-x ; x)=-\frac{1}{2[n] q}$,

(2) $D_{n, q}^{*}\left((t-x)^{2} ; x\right) \leq[1+2 \mu]_{q} \frac{x}{[n]_{q}}-\frac{1}{4[n]_{q}^{2}}\left(2[1+2 \mu]_{q}-1\right)$.

Proof For the proof of this lemma, we use Lemma 2.1. In view of

$$
D_{n}^{*}(t-x ; x)=D_{n}^{*}(t ; x)-D_{n}^{*}(1 ; x)
$$

(1) follows immediately.

Also

$$
\begin{aligned}
D_{n}^{*}\left((t-x)^{2} ; x\right)= & D_{n}^{*}\left(t^{2} ; x\right)-2 x D_{n}^{*}(t ; x)+x^{2} D_{n}^{*}(1 ; x) \\
\leq & x^{2}+\left([1+2 \mu]_{q}-1\right) \frac{x}{[n]_{q}}-\frac{1}{4[n]_{q}^{2}}\left(2[1+2 \mu]_{q}-1\right) \\
& -2 x\left(x-\frac{1}{2[n]_{q}}\right)+x^{2} \\
\leq & {[1+2 \mu]_{q} \frac{x}{[n]_{q}}-\frac{1}{4[n]_{q}^{2}}\left(2[1+2 \mu]_{q}-1\right) . }
\end{aligned}
$$

This proves (2).

\section{Main results}

We obtain the Korovkin-type approximation properties for our operators (see [20-22]).

Let $C_{B}\left(\mathbb{R}^{+}\right)$be the set of all bounded and continuous functions on $\mathbb{R}^{+}=[0, \infty)$, which is a linear normed space with

$$
\|f\|_{C_{B}}=\sup _{x \geq 0}|f(x)| .
$$


Let

$$
H:=\left\{f: x \in[0, \infty), \frac{f(x)}{1+x^{2}} \text { is convergent as } x \rightarrow \infty\right\} .
$$

Theorem 3.1 Let $D_{n, q}^{*}(\cdot ; \cdot)$ be the operators defined by (2.2). Then, for any function $f \in$ $C_{\zeta}[0, \infty) \cap H, \zeta \geq 2$,

$$
\lim _{n \rightarrow \infty} D_{n, q}^{*}(f ; x)=f(x)
$$

is uniform on each compact subset of $[0, \infty)$, where $x \in\left[\frac{1}{2}, b\right), b>\frac{1}{2}$.

Proof The proof is based on Lemma 2.1 and the well-known Korovkin theorem regarding the convergence of a sequence of linear positive operators, so it is enough to prove the conditions

$$
\lim _{n \rightarrow \infty} D_{n, q}^{*}\left(t^{j} ; x\right)=x^{j}, \quad j=0,1,2(\operatorname{as} n \rightarrow \infty)
$$

uniformly on $[0,1]$.

Clearly, $\frac{1}{[n]_{q}} \rightarrow 0(n \rightarrow \infty)$ we have

$$
\lim _{n \rightarrow \infty} D_{n, q}^{*}(t ; x)=x, \quad \lim _{n \rightarrow \infty} D_{n, q}^{*}\left(t^{2} ; x\right)=x^{2} .
$$

This completes the proof.

We recall the weighted spaces of the functions on $\mathbb{R}^{+}$, which are defined as follows:

$$
\begin{aligned}
& P_{\rho}\left(\mathbb{R}^{+}\right)=\left\{f:|f(x)| \leq M_{f} \rho(x)\right\}, \\
& Q_{\rho}\left(\mathbb{R}^{+}\right)=\left\{f: f \in P_{\rho}\left(\mathbb{R}^{+}\right) \cap C[0, \infty)\right\}, \\
& Q_{\rho}^{k}\left(\mathbb{R}^{+}\right)=\left\{f: f \in Q_{\rho}\left(\mathbb{R}^{+}\right) \text {and } \lim _{x \rightarrow \infty} \frac{f(x)}{\rho(x)}=k(k \text { is a constant })\right\},
\end{aligned}
$$

where $\rho(x)=1+x^{2}$ is a weight function and $M_{f}$ is a constant depending only on $f$. Note that $Q_{\rho}\left(\mathbb{R}^{+}\right)$is a normed space with the norm $\|f\|_{\rho}=\sup _{x \geq 0} \frac{|f(x)|}{\rho(x)}$.

Lemma 3.2 ([23]) The linear positive operators $L_{n}, n \geq 1$ act from $Q_{\rho}\left(\mathbb{R}^{+}\right) \rightarrow P_{\rho}\left(\mathbb{R}^{+}\right)$if and only if

$$
\left\|L_{n}(\varphi ; x)\right\| \leq K \varphi(x)
$$

where $\varphi(x)=1+x^{2}, x \in \mathbb{R}^{+}$and $K$ is a positive constant.

Theorem 3.3 ([23]) Let $\left\{L_{n}\right\}_{n \geq 1}$ be a sequence of positive linear operators acting from $Q_{\rho}\left(\mathbb{R}^{+}\right) \rightarrow P_{\rho}\left(\mathbb{R}^{+}\right)$and satisfying the condition

$$
\lim _{n \rightarrow \infty}\left\|L_{n}\left(\rho^{\tau}\right)-\rho^{\tau}\right\|_{\varphi}=0, \quad \tau=0,1,2 .
$$


Then, for any function $f \in Q_{\rho}^{k}\left(\mathbb{R}^{+}\right)$, we have

$$
\lim _{n \rightarrow \infty}\left\|L_{n}(f ; x)-f\right\|_{\varphi}=0 .
$$

Theorem 3.4 Let $D_{n, q}^{*}(\cdot ; \cdot)$ be the operators defined by (2.2). Then, for each function $f \in$ $Q_{\rho}^{k}\left(\mathbb{R}^{+}\right)$, we have

$$
\lim _{n \rightarrow \infty}\left\|D_{n, q}^{*}(f ; x)-f\right\|_{\rho}=0
$$

Proof From Lemma 2.1 and Theorem 3.3, for $\tau=0$, the first condition is fulfilled. Therefore,

$$
\lim _{n \rightarrow \infty}\left\|D_{n, q}^{*}(1 ; x)-1\right\|_{\rho}=0 .
$$

Similarly, from Lemma 2.1 and Theorem 3.3, for $\tau=1,2$ we have that

$$
\begin{aligned}
\sup _{x \in[0, \infty)} \frac{\left|D_{n, q}^{*}(t ; x)-x\right|}{1+x^{2}} & \leq \frac{1}{2[n]_{q}} \sup _{x \in[0, \infty)} \frac{1}{1+x^{2}} \\
& =\frac{1}{2[n]_{q}}
\end{aligned}
$$

which implies that

$$
\begin{aligned}
\lim _{n \rightarrow \infty}\left\|D_{n, q}^{*}(t ; x)-x\right\|_{\rho}=0 & \\
\sup _{x \in[0, \infty)} \frac{\left|D_{n, q}^{*}\left(t^{2} ; x\right)-x^{2}\right|}{1+x^{2}} \leq & \frac{\left|[1+2 \mu]_{q}-1\right|}{[n]_{q}} \sup _{x \in[0, \infty)} \frac{x}{1+x^{2}} \\
& +\frac{1}{4[n]_{q}^{2}}\left|[1+2 \mu]_{q}-1\right| \sup _{x \in[0, \infty)} \frac{1}{1+x^{2}} .
\end{aligned}
$$

Hence

$$
\lim _{n \rightarrow \infty}\left\|D_{n, q}^{*}\left(t^{2} ; x\right)-x^{2}\right\|_{\rho}=0 .
$$

This completes the proof.

\section{Rate of convergence}

Let $f \in C_{B}[0, \infty]$, the space of all bounded and continuous functions on $[0, \infty)$ and $x \geq$ $\frac{1}{2 n}, n \in \mathbb{N}$. Then, for $\delta>0$, the modulus of continuity of $f$ denoted by $\omega(f, \delta)$ gives the maximum oscillation of $f$ in any interval of length not exceeding $\delta>0$, and it is given by

$$
\omega(f, \delta)=\sup _{|t-x| \leq \delta}|f(t)-f(x)|, \quad t \in[0, \infty)
$$

It is known that $\lim _{\delta \rightarrow 0+} \omega(f, \delta)=0$ for $f \in C_{B}[0, \infty)$, and for any $\delta>0$ we have

$$
|f(t)-f(x)| \leq\left(\frac{|t-x|}{\delta}+1\right) \omega(f, \delta)
$$


Now we calculate the rate of convergence of operators (2.2) by means of modulus of continuity and Lipschitz-type maximal functions.

Theorem 4.1 Let $D_{n, q}^{*}(\cdot ; \cdot)$ be the operators defined by (2.2). Then, for $f \in C_{B}[0, \infty), x \geq \frac{1}{2 n}$ and $n \in \mathbb{N}$, we have

$$
\left|D_{n, q}^{*}(f ; x)-f(x)\right| \leq 2 \omega\left(f ; \delta_{n, x}\right)
$$

where

$$
\delta_{n, x}=\sqrt{[1+2 \mu]_{q} \frac{x}{[n]_{q}}-\frac{1}{4[n]_{q}^{2}}\left(2[1+2 \mu]_{q}-1\right)} .
$$

Proof We prove it by using (4.1), (4.2) and the Cauchy-Schwarz inequality. We can easily get

$$
\left|D_{n, q}^{*}(f ; x)-f(x)\right| \leq\left\{1+\frac{1}{\delta}\left(D_{n, q}^{*}(t-x)^{2} ; x\right)^{\frac{1}{2}}\right\} \omega(f ; \delta)
$$

if we choose $\delta=\delta_{n, x}$, and by applying the result (2) of Lemma 2.2, we get the result.

Remark 4.2 For the operators $D_{n, q}(\cdot ; \cdot)$ defined by (1.9) we may write that, for every $f \in$ $C_{B}[0, \infty), x \geq 0$ and $n \in \mathbb{N}$,

$$
\left|D_{n, q}(f ; x)-f(x)\right| \leq 2 \omega\left(f ; \lambda_{n, x}\right)
$$

where by [19] we have

$$
\lambda_{n, x}=\sqrt{D_{n, q}\left((t-x)^{2} ; x\right)} \leq \sqrt{[1+2 \mu]_{q} \frac{x}{[n]_{q}}}
$$

Now we claim that the error estimation in Theorem 4.1 is better than that of (4.4) provided $f \in C_{B}[0, \infty)$ and $x \geq \frac{1}{2 n}, n \in \mathbb{N}$. Indeed, for $x \geq \frac{1}{2 n}, \mu \geq \frac{1}{2 n}$ and $n \in \mathbb{N}$, it is guaranteed that

$$
\begin{aligned}
& D_{n, q}^{*}\left((t-x)^{2} ; x\right) \leq D_{n, q}\left((t-x)^{2} ; x\right), \\
& {[1+2 \mu]_{q} \frac{x}{[n]_{q}}-\frac{1}{4[n]_{q}^{2}}\left(2[1+2 \mu]_{q}-1\right) \leq[1+2 \mu]_{q} \frac{x}{[n]_{q}},}
\end{aligned}
$$

which implies that

$$
\sqrt{[1+2 \mu]_{q} \frac{x}{[n]_{q}}-\frac{1}{4[n]_{q}^{2}}\left(2[1+2 \mu]_{q}-1\right)} \leq \sqrt{[1+2 \mu]_{q} \frac{x}{[n]_{q}}} .
$$

Now we give the rate of convergence of the operators $D_{n, q}^{*}(f ; x)$ defined in $(2.2)$ in terms of the elements of the usual Lipschitz class $\operatorname{Lip}_{M}(v)$.

Let $f \in C_{B}[0, \infty), M>0$ and $0<v \leq 1$. The class $\operatorname{Lip}_{M}(v)$ is defined as

$$
\operatorname{Lip}_{M}(v)=\left\{f:\left|f\left(\zeta_{1}\right)-f\left(\zeta_{2}\right)\right| \leq M\left|\zeta_{1}-\zeta_{2}\right|^{\nu}\left(\zeta_{1}, \zeta_{2} \in[0, \infty)\right)\right\}
$$


Theorem 4.3 Let $D_{n, q}^{*}(\cdot ; \cdot)$ be the operators defined in (2.2).Then, for each $f \in \operatorname{Lip}_{M}(v)(M>$ $0,0<v \leq 1)$ satisfying (4.9), we have

$$
\left|D_{n, q}^{*}(f ; x)-f(x)\right| \leq M\left(\delta_{n, x}\right)^{\frac{v}{2}},
$$

where $\delta_{n, x}$ is given in Theorem 4.1.

Proof We prove it by using (4.9) and Hölder's inequality. We have

$$
\begin{aligned}
\left|D_{n, q}^{*}(f ; x)-f(x)\right| & \leq\left|D_{n, q}^{*}(f(t)-f(x) ; x)\right| \\
& \leq D_{n, q}^{*}(|f(t)-f(x)| ; x) \\
& \leq M D_{n, q}^{*}\left(|t-x|^{\nu} ; x\right) .
\end{aligned}
$$

Therefore,

$$
\begin{aligned}
&\left|D_{n, q}^{*}(f ; x)-f(x)\right| \leq M \frac{[n]_{q}}{e_{\mu, q}\left([n]_{q} r_{[n]_{q}}(x)\right)} \sum_{k=0}^{\infty} \frac{\left([n]_{q} r_{[n]_{q}}(x)\right)^{k}}{\gamma_{\mu, q}(k)}\left|\frac{1-q^{2 \mu \theta_{k}+k}}{1-q^{n}}-x\right|^{\nu} \\
& \leq M \frac{[n]_{q}}{e_{\mu, q}\left([n]_{q} r_{[n]_{q}}(x)\right)} \sum_{k=0}^{\infty}\left(\frac{\left([n]_{q} r_{[n]_{q}}(x)\right)^{k}}{\gamma_{\mu, q}(k)}\right)^{\frac{2-v}{2}} \\
& \quad \times\left(\frac{\left([n]_{q} r_{\left.[n]_{q}(x)\right)^{k}}\right.}{\gamma_{\mu, q}(k)}\right)^{\frac{v}{2}}\left|\frac{1-q^{2 \mu \theta_{k}+k}}{1-q^{n}}-x\right|^{\nu} \\
& \leq M\left(\frac{n}{e_{\mu, q}\left([n]_{q} r_{[n]_{q}}(x)\right)} \sum_{k=0}^{\infty} \frac{\left([n]_{q} r_{[n]_{q}}(x)\right)^{k}}{\gamma_{\mu, q}(k)}\right)^{\frac{2-v}{2}} \\
& \quad \times\left(\frac{[n]_{q}}{e_{\mu, q}\left([n]_{q} r_{[n]_{q}}(x)\right)} \sum_{k=0}^{\infty} \frac{\left([n]_{q} r_{[n]_{q}}(x)\right)^{k}}{\gamma_{\mu, q}(k)}\left|\frac{1-q^{2 \mu \theta_{k}+k}}{1-q^{n}}-x\right|^{2}\right)^{\frac{v}{2}} \\
&=M\left(D_{n, q}^{*}(t-x)^{2} ; x\right)^{\frac{v}{2}} .
\end{aligned}
$$

This completes the proof.

Let

$$
C_{B}^{2}\left(\mathbb{R}^{+}\right)=\left\{g \in C_{B}\left(\mathbb{R}^{+}\right): g^{\prime}, g^{\prime \prime} \in C_{B}\left(\mathbb{R}^{+}\right)\right\}
$$

with the norm

$$
\|g\|_{C_{B}^{2}\left(\mathbb{R}^{+}\right)}=\|g\|_{C_{B}\left(\mathbb{R}^{+}\right)}+\left\|g^{\prime}\right\|_{C_{B}\left(\mathbb{R}^{+}\right)}+\left\|g^{\prime \prime}\right\|_{C_{B}\left(\mathbb{R}^{+}\right)},
$$

also

$$
\|g\|_{C_{B}\left(\mathbb{R}^{+}\right)}=\sup _{x \in \mathbb{R}^{+}}|g(x)| .
$$


Theorem 4.4 Let $D_{n, q}^{*}(\cdot ; \cdot)$ be the operators defined in (2.2). Then for any $g \in C_{B}^{2}\left(\mathbb{R}^{+}\right)$we have

$$
\left|D_{n, q}^{*}(f ; x)-f(x)\right| \leq\left\{\left(-\frac{1}{2[n]_{q}}\right)+\frac{\delta_{n, x}}{2}\right\}\|g\|_{C_{B}^{2}\left(\mathbb{R}^{+}\right)},
$$

where $\delta_{n, x}$ is given in Theorem 4.1.

Proof Let $g \in C_{B}^{2}\left(\mathbb{R}^{+}\right)$. Then, by using the generalized mean value theorem in the Taylor series expansion, we have

$$
g(t)=g(x)+g^{\prime}(x)(t-x)+g^{\prime \prime}(\psi) \frac{(t-x)^{2}}{2}, \quad \psi \in(x, t)
$$

By applying the linearity property on $D_{n, q}^{*}$, we have

$$
D_{n, q}^{*}(g, x)-g(x)=g^{\prime}(x) D_{n, q}^{*}((t-x) ; x)+\frac{g^{\prime \prime}(\psi)}{2} D_{n, q}^{*}\left((t-x)^{2} ; x\right),
$$

which implies that

$$
\begin{aligned}
& \left|D_{n, q}^{*}(g ; x)-g(x)\right| \\
& \quad \leq\left(-\frac{1}{2[n]_{q}}\right)\left\|g^{\prime}\right\|_{C_{B}\left(\mathbb{R}^{+}\right)}+\left([1+2 \mu]_{q} \frac{x}{[n]_{q}}-\frac{1}{4[n]_{q}^{2}}\left(2[1+2 \mu]_{q}-1\right)\right) \frac{\left\|g^{\prime \prime}\right\|_{C_{B}\left(\mathbb{R}^{+}\right)}}{2} .
\end{aligned}
$$

From (4.11) we have $\left\|g^{\prime}\right\|_{C_{B}[0, \infty)} \leq\|g\|_{C_{B}^{2}[0, \infty)}$,

$$
\begin{aligned}
& \left|D_{n, q}^{*}(g ; x)-g(x)\right| \\
& \quad \leq\left(-\frac{1}{2[n]_{q}}\right)\|g\|_{C_{B}^{2}\left(\mathbb{R}^{+}\right)}+\left([1+2 \mu]_{q} \frac{x}{[n]_{q}}-\frac{1}{4[n]_{q}^{2}}\left(2[1+2 \mu]_{q}-1\right)\right) \frac{\|g\|_{C_{B}^{2}\left(\mathbb{R}^{+}\right)}}{2} .
\end{aligned}
$$

The proof follows from (2) of Lemma 2.2.

The Peetre's $K$-functional is defined by

$$
K_{2}(f, \delta)=\inf _{C_{B}^{2}\left(\mathbb{R}^{+}\right)}\left\{\left(\|f-g\|_{C_{B}\left(\mathbb{R}^{+}\right)}+\delta\left\|g^{\prime \prime}\right\|_{C_{B}^{2}\left(\mathbb{R}^{+}\right)}\right): g \in \mathcal{W}^{2}\right\},
$$

where

$$
\mathcal{W}^{2}=\left\{g \in C_{B}\left(\mathbb{R}^{+}\right): g^{\prime}, g^{\prime \prime} \in C_{B}\left(\mathbb{R}^{+}\right)\right\}
$$

There exists a positive constant $C>0$ such that $K_{2}(f, \delta) \leq C \omega_{2}\left(f, \delta^{\frac{1}{2}}\right), \delta>0$, where the second-order modulus of continuity is given by

$$
\omega_{2}\left(f, \delta^{\frac{1}{2}}\right)=\sup _{0<h<\delta^{\frac{1}{2}}} \sup _{x \in \mathbb{R}^{+}}|f(x+2 h)-2 f(x+h)+f(x)| .
$$


Theorem 4.5 For $x \geq \frac{1}{2 n}, n \in \mathbb{N}$ and $f \in C_{B}\left(\mathbb{R}^{+}\right)$, we have

$$
\begin{aligned}
& \left|D_{n, q}^{*}(f ; x)-f(x)\right| \\
& \quad \leq 2 M\left\{\omega_{2}\left(f ; \sqrt{\frac{\left(-\frac{1}{[n]_{q}}\right)+\delta_{n, x}}{4}}\right)+\min \left(1, \frac{\left(-\frac{1}{[n]_{q}}\right)+\delta_{n, x}}{4}\right)\|f\|_{C_{B}\left(\mathbb{R}^{+}\right)}\right\},
\end{aligned}
$$

where $M$ is a positive constant, $\delta_{n, x}$ is given in Theorem 4.3 and $\omega_{2}(f ; \delta)$ is the second-order modulus of continuity of the function $f$ defined in (4.15).

Proof We prove this by using Theorem 4.4

$$
\begin{aligned}
\left|D_{n, q}^{*}(f ; x)-f(x)\right| & \leq\left|D_{n, q}^{*}(f-g ; x)\right|+\left|D_{n, q}^{*}(g ; x)-g(x)\right|+|f(x)-g(x)| \\
& \leq 2\|f-g\|_{C_{B}\left(\mathbb{R}^{+}\right)}+\frac{\delta_{n, x}}{2}\|g\|_{C_{B}^{2}\left(\mathbb{R}^{+}\right)}+\left(-\frac{1}{2[n]_{q}}\right)\|g\|_{C_{B}\left(\mathbb{R}^{+}\right) .}
\end{aligned}
$$

From (4.11), clearly, we have $\|g\|_{C_{B}[0, \infty)} \leq\|g\|_{C_{B}^{2}[0, \infty)}$.

Therefore,

$$
\left|D_{n, q}^{*}(f ; x)-f(x)\right| \leq 2\left(\|f-g\|_{C_{B}\left(\mathbb{R}^{+}\right)}+\frac{\left(-\frac{1}{[n]_{q}}\right)+\delta_{n, x}}{4}\|g\|_{C_{B}^{2}\left(\mathbb{R}^{+}\right)}\right),
$$

where $\delta_{n, x}$ is given in Theorem 4.1.

By taking infimum over all $g \in C_{B}^{2}\left(\mathbb{R}^{+}\right)$and by using (4.13), we get

$$
\left|D_{n, q}^{*}(f ; x)-f(x)\right| \leq 2 K_{2}\left(f ; \frac{\left(-\frac{1}{[n]_{q}}\right)+\delta_{n, x}}{4}\right) .
$$

Now, for an absolute constant $Q>0$ in [24], we use the relation

$$
K_{2}(f ; \delta) \leq Q\left\{\omega_{2}(f ; \sqrt{\delta})+\min (1, \delta)\|f\|\right\} .
$$

This completes the proof.

\section{Conclusion}

The purpose of this paper is to provide a better error estimation of convergence by modification of the $q$-Dunkl analogue of Szász operators. Here we have defined a Dunkl generalization of these modified operators. This type of modification enables better error estimation on the interval $[1 / 2, \infty)$ if compared to the classical Dunkl-Szász operators [19]. We obtained some approximation results via the well-known Korovkin-type theorem. We have also calculated the rate of convergence of operators by means of modulus of continuity and Lipschitz-type maximal functions.

\section{Authors' contributions}

All authors of the manuscript have read and agreed to its content and are accountable for all aspects of the accuracy and integrity of the manuscript. 
Acknowledgements

The authors gratefully acknowledge the financial support from King Abdulaziz University, Jeddah, Saudi Arabia.

Received: 16 September 2016 Accepted: 30 January 2017 Published online: 07 February 2017

\section{References}

1. Bernstein, SN: Démonstration du théoréme de Weierstrass fondée sur le calcul des probabilités. Commun. Soc. Math. Kharkow 2(13), 1-2 (2012)

2. Szász, O: Generalization of S. Bernstein's polynomials to the infinite interval. J. Res. Natl. Bur. Stand. 45, 239-245 (1950)

3. Lupaş, A: A q-analogue of the Bernstein operator. In: Seminar on Numerical and Statistical Calculus, vol. 9, pp. 85-92. University of Cluj-Napoca, Cluj-Napoca (1987)

4. Phillips, GM: Bernstein polynomials based on the q-integers. Ann. Numer. Math. 4, 511-518 (1997)

5. Mursaleen, M, Ansari, KJ: Approximation of $q$-Stancu-beta operators which preserve $x^{2}$. Bull. Malays. Math. Sci. Soc. (2015). doi:10.1007/s40840-015-0146-9

6. Mursaleen, M, Khan, A: Statistical approximation properties of modified $q$-Stancu-beta operators. Bull. Malays. Math. Sci. Soc. (2) 36(3), 683-690 (2013)

7. Mursaleen, M, Khan, A: Generalized $q$-Bernstein-Schurer operators and some approximation theorems. J. Funct. Spaces Appl. 2013, Article ID 719834 (2013)

8. Mursaleen, M, Khan, F, Khan, A: Approximation properties for modified q-Bernstein-Kantorovich operators. Numer. Funct. Anal. Optim. 36(9), 1178-1197 (2015)

9. Mursaleen, M, Khan, F, Khan, A: Approximation properties for King's type modified q-Bernstein-Kantorovich operators. Math. Methods Appl. Sci. 38, 5242-5252 (2015)

10. Mursaleen, M, Khan, T, Nasiruzzaman, M: Approximating properties of generalized Dunkl analogue of Szász operators. Appl. Math. Inf. Sci. 10(6), 1-8 (2016)

11. Örkcü, M, Doğru, O: Weighted statistical approximation by Kantorovich type $q$-Szász Mirakjan operators. Appl. Math Comput. 217, 7913-7919 (2011)

12. Örkcü, M, Doğru, O: $q$-Szász-Mirakyan-Kantorovich type operators preserving some test functions. Appl. Math. Lett. 24, 1588-1593 (2011)

13. Wafi, A, Rao, N, Rai, D: Approximation properties by generalized-Baskakov-Kantorovich-Stancu type operators. Appl. Math. Inf. Sci. Lett. 4(3), 111-118 (2016)

14. Wafi, A, Rao, N: A generalization of Szász-type operators which preserves constant and quadratic test functions. Cogent Math. 3, 1227023 (2016)

15. Aral, A, Gupta, V, Agarwal, RP: Applications of q-Calculus in Operator Theory. Springer, New York (2013)

16. Rosenblum, M: Generalized Hermite polynomials and the Bose-like oscillator calculus. Oper. Theory, Adv. Appl. 73, 369-396 (1994)

17. Sucu, S: Dunkl analogue of Szász operators. Appl. Math. Comput. 244, 42-48 (2014)

18. Cheikh, B, Gaied, Y, Zaghouani, M: A q-Dunkl-classical q-Hermite type polynomials. Georgian Math. J. 21(2), 125-137 (2014)

19. İçōz, G, Çekim, B: Dunkl generalization of Szász operators via q-calculus. J. Inequal. Appl. 2015, 284 (2015)

20. Braha, NL, Srivastava, HM, Mohiuddine, SA: A Korovkin's type approximation theorem for periodic functions via the statistical summability of the generalized de la Vallée Poussin mean. Appl. Math. Comput. 228, 162-169 (2014)

21. Edely, OHH, Mohiuddine, SA, Noman, AK: Korovkin type approximation theorems obtained through generalized statistical convergence. Appl. Math. Lett. 23(11), 1382-1387 (2010)

22. Mohiuddine, SA: An application of almost convergence in approximation theorems. Appl. Math. Lett. 24(11), 1856-1860 (2011)

23. Gadzhiev, AD: The convergence problem for a sequence of positive linear operators on unbounded sets and theorems analogues to that of PP Korovkin. Sov. Math. Dokl. 15(5), 1433-1436 (1974)

24. Ciupa, A: A class of integral Favard-Szász type operators. Stud. Univ. Babeş-Bolyai, Math. 40(1), 39-47 (1995)

\section{Submit your manuscript to a SpringerOpen ${ }^{\circ}$ journal and benefit from:}

- Convenient online submission

Rigorous peer review

- Immediate publication on acceptance

- Open access: articles freely available online

- High visibility within the field

- Retaining the copyright to your article 